Methods An inner-city ED implemented an opt-in rapid oral-fluid HIV screening programme since 2005; during the summer of 2007 , HIV testing facilitators offered $24 / 7$ bedside rapid testing to patients aged 18-64 years. During the same period, an HIV seroprevalence study enrolled adult patients who gave consent for use of their blood samples. Known HIV positivity was determined by either chart review or self-report. Serum samples were tested for HIV by EIA and all positives were confirmed by Western blot followed by RNA viral load testing.

Results There were 3,884 samples, including 153 from known HIV positives for the seroprevalence study. Among the remaining 3,731 visits, 1,286(34\%) were offered bedside HIV testing; 2,445(66\%) were not. Among those offered, 693 declined, and 561 were tested (32 accepted but were never tested). Seroprevalence data revealed the following rates of undiagnosed HIV infections: $2.0 \%$ in those offered versus $5.3 \%$ in those not offered ( $p<0.001) ; 2.5 \%$ in those who declined, $0.6 \%$ in those tested, and $15.6 \%$ in those accepted but not tested $(p<0.001)$. Mean viral load was significantly higher in those not offered the screening as compared to those offered (difference: 63,441, 95\%: 3,310-123,572).

Conclusion There was a disporportinately high prevalence of undiagnosed HIV infection in ED patients who were not offered HIV screening and in those who declined screening, versus those who accepted testing. This indicates that even with an intensive established opt-in counsellor-based rapid HIV screening model, significant missed opportunities remain with regard to identifying undiagnosed HIV-infected individuals in the ED.

\section{P5.028 SWAB2KNOW: PROVIDE AN ORAL FLUID SAMPLE ON SITE, CHECK YOUR HIV TEST RESULT ONLINE}

doi:10.1136/sextrans-2013-051184.1073

${ }^{1,2} \mathbf{T}$ Platteau, ${ }^{1} \mathrm{~K}$ Fransen, ${ }^{1} \mathrm{C}$ Nöstlinger, ${ }^{1,2} \mathrm{~L}$ Albers, 'L Manirankunda, ${ }^{1} \mathrm{~T}$ Vermoesen, ${ }^{1} \mathrm{E}$ Florence. 'Institute of Tropical Medicine, Antwerp, Belgium; ${ }^{2}$ Helpcenter ITG, Antwerp, Belgium

Background Despite the high testing rate in Belgium a considerable number of individuals remain undiagnosed. Outreach and minimally invasive strategies might increase the uptake of HIVtesting. Swab2know aims at evaluating the feasibility of a noninvasive and confidential HIV test strategy among groups with a high risk of acquiring HIV infection (Subsaharan African migrants; SAM, and men who have sex with men; MSM) in community settings.

Methods Swab2know is using oral fluid samples for HIV-testing. Oral fluid is collected on a validated device $\left(\mathrm{Oracol}^{\circledR}\right)$. Serologic HIV testing (Genscreen ${ }^{\circledR} \mathrm{HIV}-1 / 2$ v2) and quality control (IgG determination) are performed at the Institute of Tropical Medicine's (ITM) AIDS Reference Lab.

Participants can choose to receive their result either through a secured website (www.swab2know.be) or personal counselling. Reactive results are disclosed as indicative for HIV-infection, needing confirmation on blood using the gold standard tests. These participants are contacted by phone after they checked their result, offering confirmation tests or referral to a local HIV-treatment centre. In case of a non-reactive test, participants are offered repeated testing after four months by ordering an oral fluid sampling device kit online. Data on age, sex, origin, HIV-testing behaviour, and sexual activity are collected using a survey.

Results After 2.5 months, 166 participants were tested in 11 settings. Fifty-three were SAM (31.9\%), 111 MSM (68.1\%). Twentytwo SAM (41.5\%), and all MSM (100\%) chose to receive their result through the website. Nine participants tested positive for HIV: three SAM (5.7\%), and 6 MSM (5.4\%).

Preliminary experiences show a high uptake, and good acceptance of sampling method, and online communication of test results.
Conclusion Preliminary experiences with this low-threshold method are promising, showing high acceptance and satisfaction with the online tool. Increasing uptake by ordering sampling devices online, is studied.

Up-to-date results and more specific conclusions will be presented at the conference.

\section{P5.029 HIV SCREENING SERVICES: IMPROVING UPTAKE IN RURAL AND HARD TO REACH POPULATION GROUPS}

doi:10.1136/sextrans-2013-051184.1074

C 0 Nwuba, A Eshalomi, A Abubakar, A Omoloja, J Adedayo. Prevention Organizational Systems, AIDS Care and Treatment project, MSH, Ilorin, Nigeria

Background Despite significant advances in HIV programme implementation, uptake of HIV screening services remains notably low in rural areas. Most HIV screening centres are concentrated in urban areas resulting in low coverage of HIV testing services in rural communities. This paper analyses the outcome of community and facility based interventions to increase access and uptake of HIV screening services in rural and hard to reach population groups with limited access in Nigeria.

Methods In order to increase uptake of HIV screening services in rural communities, the MSH PrO-ACT project in collaboration with Kwara state ministry of health implemented the following interventions

1. Community awareness campaigns to increase knowledge about HIV transmission and prevention.

2. Community mobile HIV testing programmes which utilised existing community structures such as churches, empty school rooms and other community buildings were established to provide temporary HIV screening services.

3. Capacity of selected community members was built on HIV counselling and testing in order to increase the number of HIV screening workers.

4. HIV screening services were offered on communal market days to increase accessibility and uptake.

5. Referral linkages from HIV testing points to HIV clinics was strengthened to ensure that every client who tests positive accesses care and treatment services.

6. HIV screening services were integrated into maternity, family planning and sexually transmitted infection units of rural clinics to reduce stigmatisation and increase uptake.

Results After 12 months, 12,436 (Male- 3,616, Female- 8,820) people were screened for HIV. 405 persons tested positive and 397 persons (M-176, F- 82) were linked and enrolled into HIV care and treatment programmes. Out of this, 235 persons were eligible for anti-retroviral therapy and commenced treatment.

Conclusion Existing inequalities between urban and rural areas highlights the need to adopt innovative approaches aimed at increasing uptake of HIV screening services.

\section{P5.030 NEW AVENUES FOR PROACTIVE HIV TESTING IN PRIMARY CARE REQUIRED: A TREND ANALYSIS OF TWENTY YEARS OF HIV-RELATED CONSULTATIONS IN DUTCH GENERAL PRACTISE}

doi:10.1136/sextrans-2013-051184.1075

1,2,3 E A M van Bergen, ${ }^{4} \mathrm{~S}$ Dorsman, ${ }^{3} \mathrm{~V} V \mathrm{~F}$ van den Broek, ${ }^{4}$ P Spreeuwenberg, ${ }^{4} \mathrm{G}$ Donker. 'STI AIDS The Netherlands, Amsterdam, The Netherlands; ${ }^{2}$ Department of General Practice AMC-UVA, Amsterdam, The Netherlands; ${ }^{3}$ Centre for Infectious Diseases, RIVM, Bilthoven, The Netherlands; ${ }^{4}$ NIVEL, Utrecht, The Netherlands

Background In the Netherlands $30-40 \%$ of HIV-infected persons are not aware of their status. Half of the newly diagnosed present late. Late treatment reduces life expectancy with approximately 10 years. Early treatment reduces transmission to sex partners with $96 \%$. A more proactive role in testing in primary care is advocated. 